Psychother Psychosom 2009;78:385-387

DOI: $10.1159 / 000235982$

\section{Childhood Sexual Abuse and Personality Differentiating High and Low Alexithymia in a Depressed Population}

Raluca A. Topciu a, b, Xi Zhao c, Wan Tang c, Marnin J. Heiseld, Nancy L. Talbot ${ }^{\mathrm{d}}$, Paul R. Duberstein ${ }^{\mathrm{b}}$

${ }^{a}$ Department of Clinical and Social Sciences in Psychology, University of Rochester, ${ }^{b}$ Laboratory of Personality and Development, Department of Psychiatry, ' Department of Biostatistics and Computational Psychology, and ${ }^{\mathrm{d}}$ Department of Psychiatry, University of Rochester Medical Center, Rochester

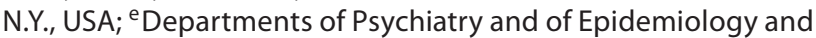
Biostatistics, Schulich School of Medicine and Dentistry, The University of Western Ontario, London, Ont., Canada

Alexithymia, a clinical condition typified by a reported inability to identify or describe one's emotions, is associated with various forms of psychopathology, including depression. Using cluster analyses, Vanheule et al. [1] identified two groups of depressed outpatients, i.e. highly and moderately alexithymic. Highly alexithymic (HA) outpatients were more likely to be female, less likely to have children and were characterized by more somatic-affective symptoms of depression and interpersonal aloofness.

We extended these findings by examining personality traits and childhood sexual abuse history. In light of prior research, we expected that higher levels of neuroticism, lower levels of extraversion and openness to experience $[2,3]$ and a greater prevalence of childhood sexual abuse $[4,5]$ would characterize the HA group. Participants were 94 depressed patients [57.45\% with recurrent major depressive disorder (MDD), 37.23\% with single-episode MDD, 5.32\% with depressive disorder not otherwise specified] 50 years of age and older recruited from psychiatric treatment facilities in Upstate New York.

Age-eligible patients who were thought to have an affective disorder were invited to participate. Individuals providing written informed consent completed questionnaires and the Structured Clinical Interview for DSM-IV Axis I disorders [6]. Diagnoses were made in consensus conferences following reviews of the interview and medical chart data. The validity of this diagnostic method is well established [7].

Alexithymia was assessed with the 20-item self-report Toronto Alexithymia Scale [8]. Its 3 subscales measure difficulty identifying feelings and distinguishing them from bodily sensations (DIF), difficulty describing and communicating feelings (DDF) and externally oriented thinking (EOT), the latter being a tendency to focus on concrete details of external events rather than on aspects of inner experience. Depressive symptom severity was assessed with the Beck Depression Inventory-II (BDI-II) [9]. Three BDI-II subscales measure the cognitive, affective and somatic symptoms of depression [10, 11]. Five personality domains, i.e. neuroticism, extraversion, openness to experience, agreeableness and conscientiousness, were assessed with the NEO Personality Inventory [12]. History of childhood sexual abuse was assessed using the Childhood Sexual Abuse subscale of the Childhood Trauma Questionnaire [13].
A latent class cluster analysis (M-Plus 4.20) [14] was performed on the DIF, DDF and EOT subscales. Cluster solutions were compared based on the Bayesian information criterion, Akaike's information criterion and entropy. A better model fit is indicated by lower values of the Bayesian information criterion and Akaike's information criterion, and a better classification is indicated by entropy values closer to 1 . All 3 indicators favored a 3 -cluster solution. This solution identified 3 groups, i.e. low alexithymia (LA; $\mathrm{n}=11,63.64 \%$ women), moderate alexithymia (MA; $\mathrm{n}=40,60 \%$ women $)$ and HA ( $n=43,60.47 \%$ women). Table 1 presents descriptive statistics for our study population. The distribution of mood diagnoses, single-episode MDD, recurrent MDD and depressive disorder not otherwise specified was not significantly different among the 3 alexithymic clusters ( $\chi^{2}$ test $\left.5.89, \mathrm{p}>0.05\right)$.

We conducted 10 separate multivariate generalized logit regressions; odds ratios were calculated for LA versus HA and MA versus HA class membership. Putative predictors were total BDIII and the 3 subscales, childhood sexual abuse and the 5 personality domains. Covariates were age, gender and education. The 3 BDI-II subscales as well as the total score significantly distinguished LA and MA from HA. Lower levels of depressive symptoms significantly decreased the odds of HA membership. Odds ratios ranged between 0.32 and $0.94(\mathrm{p}<0.05)$.

Childhood sexual abuse distinguished MA from HA; lower levels of childhood sexual abuse decreased the odds of HA membership (odds ratio 0.60, confidence interval 0.38-0.96; $\mathrm{p}<0.05$ ). Neuroticism, openness and conscientiousness also distinguished the 3 groups. Low neuroticism decreased the odds of HA membership, with odds ratios ranging between 0.95 and $0.97(\mathrm{p}<0.01)$. Low openness and conscientiousness increased the odds of HA membership, with odds ratios ranging between 1.02 and 1.07 ( $\mathrm{p}<$ 0.05).

The present study is limited by the small sample size. Findings cannot be generalized to other demographic groups. The crosssectional design precludes causal conclusions.

Our findings partially replicated the results of Vanheule et al. [1]; however, while 3 groups emerged in our cluster analyses, 2 groups emerged in theirs. However, they generated 3 clusters in an initial solution based on the DIF, DDF and EOT subscales, and a 2-cluster solution emerged when they excluded EOT from the analysis because of lack of differentiation. In our analysis, we selected the cluster solution based on all 3 subscales because EOT did distinguish the groups. Like Vanheule et al. [1], we found that the HA group was characterized by more depressive symptoms. In contrast to their results, we did not find gender differences in group composition.

The HA group in our study was characterized by higher neuroticism and lower openness to experience and conscientiousness, a profile that reflects a distressed personality type [15-17], which has been related to poorer health outcomes and general functioning and more psychological distress [18]. A similar personality profile was observed for the MA group, with the notable exception being their above-average levels of agreeableness and openness to experience.

Interestingly, the HA group was characterized by more childhood sexual abuse compared to the MA patients. These findings add to the mounting evidence for a relationship between childhood sexual abuse and alexithymia $[4,5,19]$; individuals with a history of childhood sexual abuse may have a reduced capacity to experience emotion in relation to their trauma, and this phenom- 
Table 1. Descriptive statistics

\begin{tabular}{|c|c|c|c|}
\hline & $\begin{array}{l}\text { LA group } \\
(\mathrm{n}=11)\end{array}$ & $\begin{array}{l}\text { MA group } \\
(\mathrm{n}=40)\end{array}$ & $\begin{array}{l}\text { HA group } \\
(\mathrm{n}=43)\end{array}$ \\
\hline Age, years & $58.64 \pm 6.87$ & $60.95 \pm 8.67$ & $59.21 \pm 10.00$ \\
\hline Education, years & $14.00 \pm 2.28$ & $13.65 \pm 2.96$ & $12.74 \pm 2.62$ \\
\hline \multicolumn{4}{|l|}{ TAS-20 } \\
\hline DIF & $9.36 \pm 2.46$ & $15.80 \pm 4.38$ & $25.53 \pm 4.18$ \\
\hline DDF & $7.81 \pm 2.14$ & $13.25 \pm 2$ & 2.63 \\
\hline EOT & $10.00 \pm 3.71$ & $16.13 \pm 3.39$ & $18.44 \pm 3.60$ \\
\hline Total & $27.18 \pm 4.47$ & $45.18 \pm 4.91$ & \\
\hline \multicolumn{4}{|l|}{ BDI-II } \\
\hline Cognitive & $2.50 \pm 3.06$ & $5.45 \pm 4.25$ & $7.36 \pm 4.63$ \\
\hline Affective & $1.18 \pm 1.25$ & $3.60 \pm 2.33$ & $4.47 \pm 2.62$ \\
\hline Somatic & $4.55 \pm 3.50$ & $6.25 \pm 3.71$ & $8.74 \pm 4.35$ \\
\hline Total & $12.18 \pm 10.19$ & $21.05 \pm 12.52$ & $28.63 \pm 14.40$ \\
\hline \multicolumn{4}{|l|}{ Childhood sexual } \\
\hline abuse & $9.45 \pm 7.33$ & $7.20 \pm 4.64$ & $10.28 \pm 6.46$ \\
\hline \multicolumn{4}{|l|}{ NEO-PI-R } \\
\hline Neuroticism & $63.25 \pm 28.92$ & $78.31 \pm 27.99$ & $89.50 \pm 20.92$ \\
\hline Extraversion & $55.99 \pm 18.24$ & $43.46 \pm 32.45$ & $36.66 \pm 24.11$ \\
\hline Openness & $74.68 \pm 35.97$ & $57.04 \pm 30.57$ & $39.67 \pm 27.17$ \\
\hline Agreeableness & $54.77 \pm 32.68$ & $69.12 \pm 30.47$ & $52.45 \pm 29.18$ \\
\hline Conscientiousness & $56.35 \pm 25.94$ & $50.76 \pm 29.94$ & $37.76 \pm 25.69$ \\
\hline \multicolumn{4}{|c|}{$\begin{array}{l}\text { Data are shown as means } \pm \text { SD. Education is measured as a } \\
\text { continuous variable in number of years. Childhood sexual abuse } \\
\text { was measured using the Childhood Sexual Abuse subscale of the } \\
\text { Childhood Trauma Questionnaire, for which the following cutoff } \\
\text { points have been established: } 5 \text { or lower = no abuse; } 6-7=\text { low abuse; } \\
8-12=\text { moderate abuse; } 12 \text { or higher }=\text { severe abuse [13]. Standard } \\
\text { scores for the NEO Personality Inventory (NEO-PI-R) are present- } \\
\text { ed, to facilitate interpretation. Raw scores were used in analyses. } \\
\text { TAS- } 20=\text { Toronto Alexithymia Scale. }\end{array}$} \\
\hline
\end{tabular}

enon may generalize to experiencing all emotions. Childhood sexual abuse has been found to interfere with the development of emotion regulation [20] and to be related to attachment disturbance [21]. The combination of childhood sexual abuse and alexithymia must be considered in the design and implementation of treatment studies, as these patients are more resistant to treatment (the attachment disturbance makes it more difficult for these clients to engage in a therapeutic relationship) and have slower recovery rates and poorer outcomes [4, 22, 23].

These findings emphasize the importance of considering whether a patients is alexithymic. Identifying a patient as alexithymic may suggest a history of early traumatic events which increases the likelihood and severity of depression. Treatment should be tailored to address the depressive symptoms along with the affective experience (identification, differentiation, labeling and management of feelings).

\section{Acknowledgement}

This study was supported by grants R01MH60285, K24MH072712 and R24AG031089 from the National Institutes of Health, USA.

\section{References}

$\rightarrow 1$ Vanheule S, Desmet M, Verhaeghe P, Bogaerts S: Alexithymic depression: evidence for a depression subtype? Psychother Psychosom 2007; 77:61-62.

-2 Luminet O, Bagby RM, Wagner H, Taylor GJ, Parker JDA: Relation between alexithymia and the five-factor model of personality: a facetlevel analysis. J Pers Assess 1999;73:345-358.

-3 Zimmermann G, Rossier J, Meyer de Stadelhofen F, Gaillard F: Alexithymia assessment and relations with dimensions of personality. Eur J Psychol Assess 2005;21:23-33.

$\checkmark 4$ Hund AR, Espelage DL: Childhood sexual abuse, disordered eating, alexithymia, and general distress: a mediational model. J Couns Psychol 2005;52:559-573.

5 McLean LM, Toner B, Jackson J, Desrocher M, Stuckless N: The relationship between childhood sexual abuse, complex post-traumatic stress disorder and alexithymia in two outpatient samples: examination of women treated in community and institutional clinics. J Child Sex Abus 2006;15:1-17.

6 First MB, Spitzer RL, Gibbon M, Williams JBW: Structured Clinical Interview for DSM-IV Axis I Disorders - Patient Edition, version 2.0, 4/97 revision. New York, Biometrics Research Department, New York Psychiatric Institute, 1997.

7 Conner KR, Duberstein PR, Conwell Y: The validity of proxy-based data in suicide research: a study of patients 50 years of age and older who attempted suicide. I. Psychiatric diagnoses. Acta Psychiatr Scand 2001;104:204-209.

8 Bagby RM, Parker JDA, Taylor GJ: The twenty-item Toronto Alexithymia Scale. I. Item selection and cross-validation of the factor structure. J Psychosom Res 1994;38:23-32.

9 Beck AT, Steer RA, Brown GK: Beck Depression Inventory - Second Edition Manual. San Antonio, Psychological Corporation, 1996.

10 Vanheule S, Desmet M, Groenvynck H, Rosseel Y, Fontaine J: The factor structure of the Beck Depression Inventory-II: an evaluation. Assessment 2008;15:177-187.

11 Buckley TC, Parker JD, Heggie J: A psychometric evaluation of the BDIII in treatment seeking substance abusers. J Subst Abuse Treat 2001;20: 197-204.

12 Costa PT, McCrae RR: Revised NEO Personality Inventory (NEO-PIR) and NEO Five-Factor Inventory (NEO-FFI). Odessa, Psychological Assessment Resources, 1992.

13 Bernstein DP, Fink L, Handelsman L, Foote J, Lovejoy M, Wenzel K, Sapareto E, Ruggiero J: Initial reliability and validity of a new retrospective measure of child abuse and neglect. Am J Psychiatry 1994;151: 1132-1136.

14 Muthen B, Muthen LK: M+ User's Guide, ed 4. Los Angeles, Muthen \& Muthen, 2006.

15 Chapman BP, Duberstein PR, Lyness JM: The distressed personality type: replicability and general health associations. Eur J Pers 2007;21: 911-929.

16 Claes L, Vandereycken W, Luyten P, Soenens B, Pieters G, Vertommen $\mathrm{H}$ : Personality prototypes in eating disorders based on the Big Five model. J Pers Disord 2006;20:401-416.

17 Westen D, Harnden-Fischer J: Personality profiles in eating disorders: rethinking the distinction between axis I and axis II. Am J Psychiatry 2001;158:547-562.

18 Denollett J, Conraads VM, Brutsaeri DL, DeClerck LS, Stevens WJ, Vrints CJ: Cytokines and immune activation in systolic heart failure: the role of type D personality. Brain Behav Immun 2003;7:304-309.

19 Bermond B, Moormann PP, Albach F, van Dijke A: Impact of severe childhood sexual abuse on the development of alexithymia in adulthood. Psychother Psychosom 2008;77:260-262.

20 Shields AM, Ryan RM, Cichetti D: The development of emotional and behavioral self-regulation and social competence among maltreated school-age children. Dev Psychopathol 1994;6:57-75.

21 Bailey HN, Moran G, Pederson DR: Childhood maltreatment, complex trauma symptoms, and unresolved attachment in an at-risk sample of adolescent mothers. Attach Hum Dev 2007;9:139-161. 
22 Scher D, Twaite JA: The relationship between child sexual abuse and alexithymic symptoms in a population of recovering adult substance abusers. J Child Sex Abus 1999;8:25-40.

23 Zeitlin SB, McNally RJ, Cassiday KL: Alexithymia in victims of sexual assault: An effect of repeated traumatization? Am J Psychiatry 1993; 150:661-663.

Raluca A. Topciu, MD, MA

Department of Psychiatry, University of Rochester Medical Center 300 Crittenden Boulevard, Rochester, NY 14642 (USA)

Tel. +1 585275 6671, Fax +1 5852731384

E-Mail raluca_topciu@urmc.rochester.edu

Psychother Psychosom 2009;78:387-390

DOI: $10.1159 / 000235983$

\section{Well-Being Therapy in Children with Emotional and Behavioral Disturbances: A Pilot Investigation}

\section{Elisa Albieri, Dalila Visani, Emanuela Offidani, Fedra Ottolini, Chiara Ruini}

Affective Disorders Program and Laboratory of Experimental Psychotherapy, Department of Psychology, University of Bologna, Bologna, Italy

Anxiety, mood disorders and somatoform disorders are among the most prevalent forms of psychological suffering during childhood and adolescence. They are often associated with poor academic performance [1] and a number of negative outcomes; furthermore, if untreated, these problems can be predictors of severer anxiety and mood disorders in adulthood [2, 3]. Brent [4] has underlined how, even in the best center with the best clinicians, only $37 \%$ of the depressed adolescents involved have remitted. This highlights the need for new treatment modalities, which focus on each child's competencies and the development of optimal functioning in youth $[5,6]$.

A specific psychotherapeutic strategy for enhancing well-being (well-being therapy; WBT) [7] has been developed from the multidimensional model of psychological well-being by Ryff [8]. In controlled trials on adult patients with affective disorders, WBT was found to be effective in determining remission of symptoms, improving psychological well-being and preventing episodes of relapse [9-12].

The aim of this study was to apply a modified form of WBT to a child population of patients with mood, anxiety and conduct disorders, and to test its effects in reducing symptoms and in improving new skills and competencies in children. Here, we report 4 of 14 cases who underwent this new treatment protocol, and are particularly interesting from a clinical point of view.

Four male subjects, referred to a tertiary neuropsychiatric clinic, and aged from 8 to 11 years (mean $=9.50 ; S D=1.72$ ) with different diagnoses according to DSM-IV criteria (1 oppositional-defiant disorder, $1 \mathrm{ADHD}, 1$ major depressive disorder and 1 generalized anxiety disorder; GAD) were enrolled in this study. The child

The authors have no conflicts of interest to declare. with GAD also manifested comorbidity with a learning disorder (dyslexia). None of these children were receiving pharmacological treatment, but 2 of them had a special tutor for helping them in school activities (the one with dyslexia and the one with ADHD).

Intake diagnoses were established by 2 clinical psychologists (C.R., F.O. - who were not involved in the treatment) using the Schedule for Affective Disorders and Schizophrenia for SchoolAge Children - Present and Lifetime Version (K-SADS-PL) [13]. Exclusion criteria were: diagnosis of pervasive developmental disorders and mental retardation. WBT intervention is described in table 1 and was performed by 2 clinical psychologists (E.A., D.V.). At the end of the WBT child intervention and at follow-up, each patient's clinical status was evaluated by the 2 clinical psychologists (C.R., F.O.) who established the intake diagnoses (and who were not involved in the treatment) using Kellner's global rating scale of improvement [14] and again using the K-SADS-PL [13].

Written informed consent was obtained after the procedures were explained to the children and their parents.

Case Reports

Case 1. 'A' was a 10-year-old boy who was referred to the tertiary neuropsychiatric clinic for a learning disorder (dyslexia) 1 year previously. He was also diagnosed with GAD, which was characterized by many physiological symptoms and some phobic avoidant behaviors. He was instructed to keep a daily diary, and this was associated with an improvement in his learning disorder, according to parents' and teachers' reports. At the end of the treatment, he was rated 'much better' according to Kellner's global rating scale of improvement [14], with a partial remission of GAD symptoms, particularly somatic complaints and avoidant behaviors. At follow-up, the improvements were maintained and school performance improved so much that teachers decided to dismiss the child's tutor. However, in the same period of time, his parents decided to break up and divorce and A's grandfather died. These negative events led to a new increase in A's anxiety, together with aggressive behavior towards schoolmates. He was then enrolled with his parents into a family psychotherapeutic intervention.

Case 2. 'B' was a 9-year-old boy who was referred to the tertiary neuropsychiatric clinic by his school teacher because he often had extended bouts of crying in class and had refused to go to school since 1 year. At the intake of this study, he was also diagnosed with major depressive disorder, low mood, apathy, isolation, frequent crying, thoughts of death, memory and concentration difficulties, and many somatic complaints. At the beginning of the WBT intervention, B was very reluctant to participate in the proposed activities, and appeared to be isolated. These problems were gradually overcome during the sessions. A good relationship with the therapist was established. At the end of the treatment, he was rated 'better' according to Kellner's global rating scale of improvement [14], particularly when considering mood, apathy and isolation. He improved in his autonomy and passive behaviors, according to his teachers. At follow-up, the child still presented depressive symptoms, but the positive results were maintained and somatic symptoms dramatically decreased.

Case 3. 'C' was an 8-year-old boy who was referred to the tertiary neuropsychiatric clinic by his school teacher, and received a diagnosis of combined ADHD disorder 6 months previously. At the beginning of the WBT intervention, it was difficult for the therapist to engage him in the treatment because of his attentive and behavioral deficits, which greatly improved over the course 